\title{
Um Objeto de Aprendizagem para a Disciplina de Sociologia no Ensino Médio
}

\author{
Vinicius Hartmann Ferreira - IFRS - vinihf@gmail.com \\ Vanessa Petró - IFRS - vanessapetro@gmail.com \\ Luís Guilherme Eich - IFRS - gui.eich@ @otmail.com
}

Resumo: A disciplina de Sociologia vem se consolidando no currículo do Ensino Médio. Dentre as dificuldades de desempenho dos estudantes nesta disciplina estão problemas com o pensamento abstrato, necessário para a compreensão dos conteúdos, e a escassez de recursos didáticos interativos que permitam ilustrar os conteúdos. Neste contexto, este artigo apresenta um Objeto de Aprendizagem, com metadados descritos através do padrão OBAA-Lite, que tem por objetivo auxiliar os alunos a organizar o pensamento em relação aos pensadores clássicos da Sociologia. O Objeto de Aprendizagem foi validado com 40 estudantes de $1^{\circ}$ ano do Ensino Médio e com a professora de Sociologia das turmas. A partir da validação foi possível concluir que o recurso foi bem recebido e representa um recurso digital interativo relevante para o ensino de Sociologia no Ensino Médio.

Palavras-chave: ensino de sociologia, objeto de aprendizagem, Ensino Médio

\section{A Learning Object for the Discipline of Sociology in High School}

Abstract: The discipline of Sociology has been consolidating in the curriculum of Highschool education. Among the difficulties of student performance in this course are problems with abstract thinking, needed for their understanding, and the lack of interactive educational resources to illustrate the content. In this context, this paper presents a Learning Object, described via metadata standard OBAA-Lite, which aims to help students organize thinking in relation to the classical thinkers of sociology. The Learning Object was validated with a group of 40 students from High-school education and teacher of Sociology's class. From the validation we concluded that the Learning Object was well received and represents a significant interactive digital resource for teaching Sociology in High-school.

Keywords: teaching sociology, learning object, high-school education

\section{Introdução}

Um dos entraves encontrados nos processos de ensino e de aprendizagem é a dificuldade de abstração que os estudantes apresentam. No Ensino Médio, os estudantes já atingiram uma idade em que o desenvolvimento cognitivo deveria ter alcançado a fase da abstração (Piaget, 1972). Entretanto, essa capacidade ainda se apresenta como um dos empecilhos para que os estudantes tenham um melhor desempenho, por exemplo, em componentes curriculares como a Sociologia, que pressupõe constantemente o exercício de compreender conceitos - fazer abstrações - e relacioná-los a situações reais ou partir de situações cotidianas e associá-las a determinados conceitos. Esta constatação empírica, observada na experiência com as aulas de Sociologia, forneceu subsídios para o desenvolvimento do Objeto de Aprendizagem (OA) aqui apresentado. 
Portanto, é neste contexto que Objetos de Aprendizagem podem se constituir como um recurso interessante para o ensino de Sociologia no nível médio. Entre os conteúdos previstos para esta disciplina estão muitos conceitos que precisam ser relacionados com o mundo objetivo, além de acontecimentos históricos e pensadores que contribuíram para a formação da Sociologia enquanto ciência, o que também é conteúdo a ser trabalhado em sala de aula.

Um OA é qualquer recurso digital que tenha conteúdo educacional e propósito de aprendizagem e que possa ser usado e reutilizado em diferentes contextos (Medeiros e Munhoz, 2012; Silveira e Carneiro, 2012; Wiley, 2000). Para que um OA possa ser indexado, recuperado e reutilizado a partir de um repositório digital é necessário que ele seja descrito através de metadados, que podem ser definidos como dados sobre dados (Wiley, 2000). Este trabalho utiliza o padrão OBAA-Lite para descrever os metadados do OA desenvolvido. O OBAA-Lite é uma versão simplificada do padrão OBAA que contém menos elementos a serem preenchidos (Silva, 2011; Bez et al., 2010).

O conteúdo escolhido para a operacionalização do OA aqui apresentado refere-se a pensadores importantes para a Sociologia Clássica e, portanto, relacionados à história da Sociologia. Trata-se apenas de um dos muitos conteúdos abordados por esta disciplina e também um conteúdo que pode apresentar maiores dificuldades para ser compreendido pelos estudantes, sobretudo no que se refere às diferenciações entre os pensadores que contribuíram para o surgimento da Sociologia e também as associações entre acontecimentos históricos e sociais e a produção desses pensadores em relação às interpretações da sociedade, ou seja, as implicações existentes entre acontecimentos históricos relevantes e seus resultados para a leitura da sociedade.

Tendo em vista esse conteúdo, a proposta de uma linha do tempo para situar pensadores cronologicamente tem o objetivo de auxiliar os estudantes a organizarem o pensamento e os estudos no que concerne à aprendizagem deste conteúdo de forma interativa e digital, o que também permite o compartilhamento do recurso entre diferentes professores.

\section{0 ensino de Sociologia no Nível Médio}

A disciplina de Sociologia já passou por inúmeras idas e vindas no currículo do Ensino Médio desde os fins do século XIX quando foi inserida pela primeira vez, através do decreto $n^{\circ} .981$ de 8 de novembro de 1890 (Liedke Filho, 2005). Em 1971, período da ditadura militar no Brasil, a lei 5.692 retirou pela última vez a obrigatoriedade da disciplina no currículo escolar, voltando a ser reinserida em 2008, a partir do momento em que foi sancionada a lei 11.684 (Stempkowski, 2010).

O ensino de Sociologia no nível médio enfrenta inúmeros desafios, os quais podem ser apontados como a precária formação dos professores, incluindo que de maneira recorrente os titulares da disciplina não têm formação na área de Ciências Sociais; a alta carga horária dos professores da rede pública estadual e os baixos salários. Esses problemas podem refletir em dificuldades para abordar os temas específicos da disciplina, para escolher metodologias adequadas e em relação à forma como a importância deste componente curricular é apresentada à comunidade escolar (Pereira, 2009).

Além destes aspectos, vale salientar uma cultura de desvalorização da Sociologia pelos alunos e demais membros da comunidade escolar, evidenciado em colocações que expressam que a disciplina não reprova (Pereira, 2009). A falta de material didático 
adequado não deixa de ser apontada quando o assunto é Sociologia no Ensino Médio, embora existam livros didáticos inclusive distribuídos nas escolas pelo Programa Nacional do Livro Didático ${ }^{1}$ e sites com material de apoio ${ }^{2}$.

Além de objetivos para a disciplina, os documentos do Ministério da Educação indicam temáticas que devem compor o currículo. Mas, ainda assim, a construção do currículo de Sociologia nas escolas também enfrenta desafios, pois muitas vezes eles são inadequados, em outras situações os professores não os seguem ou se sentem desorientados em relação aquilo que precisa ser ensinado (Pereira, 2007).

A Sociologia cumpre no Ensino Médio um papel que é o de desenvolver nos estudantes a imaginação sociológica, nos termos descritos por Mills (1969), na medida em que permite instigar os estudantes a decifrar uma série de conceitos sociológicos que estão presentes no cotidiano e também relacionar os conceitos com a vida concreta. Essa postura leva a um distanciamento de explicações a partir da perspectiva do senso comum (Pereira, 2007). Na medida em que Pereira (2007) enfatiza a necessidade de abordar os conteúdos de Sociologia de maneira contextualizada com a realidade dos alunos, a autora defende também a ideia de que os autores clássicos da Sociologia não se constituam como um conteúdo ministrado de maneira pura na disciplina, mas que sejam utilizados para a compreensão dos fenômenos que circundam a realidade dos estudantes.

As metodologias de ensino são variadas e é salutar que assim o sejam, contando que se coloquem de forma coerente dentro da proposta de ensino adotada por cada professor e que não se perca de vista a seriedade necessária na abordagem dos conteúdos, nem se sobrevalorize a forma em detrimento dos próprios conteúdos necessários para que os estudantes possam compreender o contexto social onde estão inseridos. Associados ao ensino de Sociologia, alguns procedimentos metodológicos são mais recorrentes, tais como aulas expositivas e dialogadas, seminários, debates, pesquisas sociológicas e também a utilização de recursos audiovisuais como filmes ou ainda textos, imagens, charges e músicas. Entretanto, o que não se pode perder de vista é que recursos, especialmente filmes, precisam ser contextualizados e o professor precisa ainda promover o estranhamento e a desnaturalização do conteúdo (Brasil, 2006).

\section{Metodologia}

A ideia para o desenvolvimento deste OA surgiu de reuniões realizadas entre docentes da área de Informática e de Sociologia de uma escola com curso técnico integrado ao ensino médio e de um aluno bolsista de nível médio vinculado a um projeto de pesquisa $^{3}, \operatorname{logo}$ após se identificar que um OA poderia contribuir para o ensino da Sociologia no Ensino Médio, através dos relatos da professora sobre as dificuldades dos estudantes e também do bolsista que já foi aluno dessa disciplina. A definição do conteúdo considerou um dos tópicos do componente curricular em que os alunos ao longo dos anos

\footnotetext{
${ }^{1}$ Não se discutirá neste artigo a respeito da qualidade dos livros

${ }^{2}$ Podem ser apontados sites como o LAVIECS, da Universidade Federal do Rio Grande do Sul; o LABES, da Universidade Federal do Rio de Janeiro; o LEFIS, da Universidade Federal de Santa Catarina e da SED/SC; o Grupo de Apoio ao Ensino de Sociologia, da Universidade de Londrina, além de muitos sites de instituições que trazem informações sobre questões sociais que compõem o currículo de sociologia.

${ }^{3} \mathrm{O}$ desenvolvimento de Objetos de Aprendizagem no padrão OBAA para as disciplinas de Biologia e Sociologia.
} 
vêm apresentando maiores dificuldades, isto é, a história da Sociologia e os pensadores clássicos importantes para a sua consolidação.

Após a definição do conteúdo que seria abordado, foi projetada a dinâmica mais adequada para tratá-lo, estabelecendo então que o OA abordaria os pensadores clássicos relacionados ao surgimento da Sociologia e suas principais contribuições, contextualizando-os historicamente através de uma linha do tempo.

Com o conteúdo e a dinâmica do OA definidos, o próximo passo foi estabelecer em qual linguagem de programação ele seria desenvolvido. Como o aluno bolsista vinculado ao projeto possuía maior experiência com a linguagem de programação Java, ela foi a escolhida. A escolha pela linguagem Java também se mostrou adequada pelo fato de ela ser portável, ou seja, um software desenvolvido em Java pode ser executado em computadores com diferentes sistemas operacionais sem qualquer alteração, desde que eles possuam a Máquina Virtual Java instalada.

\section{$4 \mathrm{O}$ desenvolvimento de uma linha do tempo interativa}

O OA desenvolvido consiste em relacionar os pensadores clássicos da sociologia com suas principais contribuições para essa área do conhecimento de forma cronológica em uma linha do tempo. Ao iniciar o OA solicita-se para o usuário qual intervalo de tempo (em anos) deve ser abrangido, informação que é fornecida pelo professor da disciplina. A partir dessa seleção, a linha do tempo e as caixas com o nome dos pensadores e suas contribuições são montadas dinamicamente e então apresentadas ao usuário distribuídas de forma aleatória.

O usuário deve arrastar as caixas até a linha do tempo relacionando um autor com a sua principal contribuição para a sociologia (Figura 1). Como a linha do tempo pode compreender um grande intervalo de tempo, ela pode ser movimentada horizontalmente tanto com o movimento do mouse quanto através dos indicadores de "próximo" e "anterior" disponíveis no canto inferior esquerdo da tela.

Quando a linha do tempo é movimentada, as caixas que não estão ainda acopladas a ela não se movimentam. Para que seja possível levar as caixas é necessário adicioná-las em uma área de transporte (1), através da qual as caixas se movem junto com o movimento da linha do tempo. Além disso, para orientar os usuários, ao clicar em uma caixa que se refere a uma contribuição de um pensador, uma breve explicação é apresentada (2).

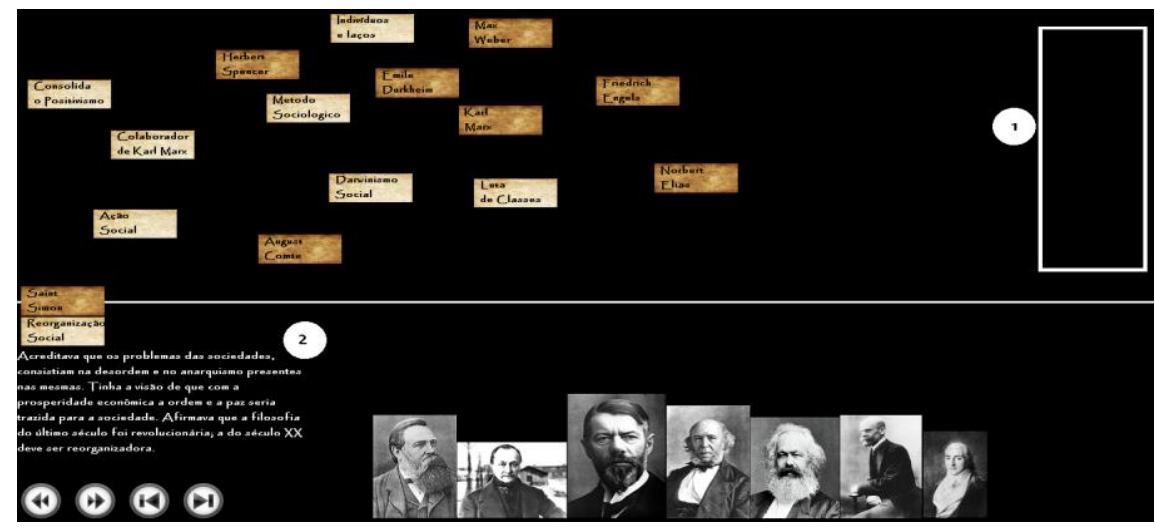

Figura 1 - Tela principal do OA desenvolvido. 
O OA foi desenvolvido em duas modalidades distintas, sendo uma delas na forma de atividade e outra na forma de avaliação. Na modalidade de atividade, ao iniciar o OA é solicitado ao usuário que indique qual será o ano de início e qual será o ano final de sua linha do tempo e ao finalizar o uso do OA, através do clique no botão "Finalizar", é apresentado para o usuário quais foram seus erros (caixas na cor vermelha) e quais foram seus acertos (caixas na cor verde) (Figura 2).
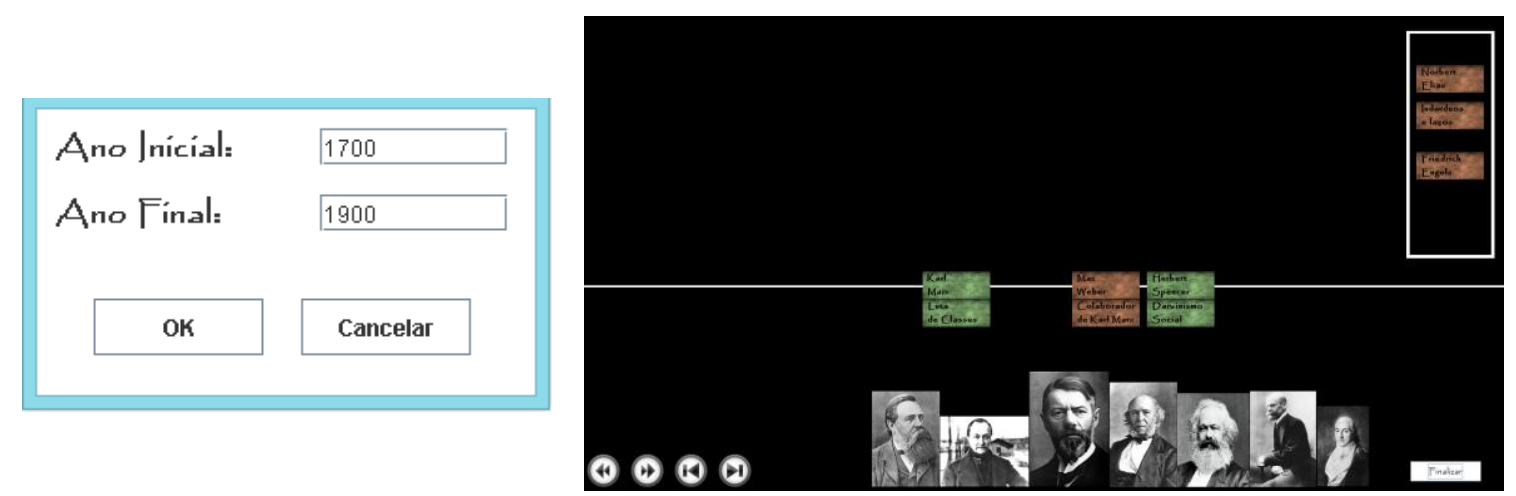

Figura 2 - (a) Tela inicial do OA na qual se deve informar o intervalo de tempo abrangido pela linha do tempo; (b) módulo de atividade do OA.

Na modalidade de avaliação, com o objetivo de permitir que o OA seja utilizado como instrumento avaliativo, ao iniciá-lo solicita-se que o usuário informe o ano de início e o de fim da linha do tempo, o peso atribuído à avaliação e o nome do aluno. Orienta-se que o peso da avaliação e o intervalo de tempo deve ser pré-definido pelo docente quando utilizar o OA nesta modalidade. Ao concluir a utilização do OA será apresentada a pontuação obtida para o usuário. O peso total da avaliação é distribuído pelo número de combinações corretas feitas, pelo estudante, entre o pensador disponível e a contribuição do mesmo para a Sociologia. Esta pontuação é armazenada, junto com as informações definidas no início da utilização do OA e o horário de início e de finalização da atividade, em um arquivo XML (Figura 3).
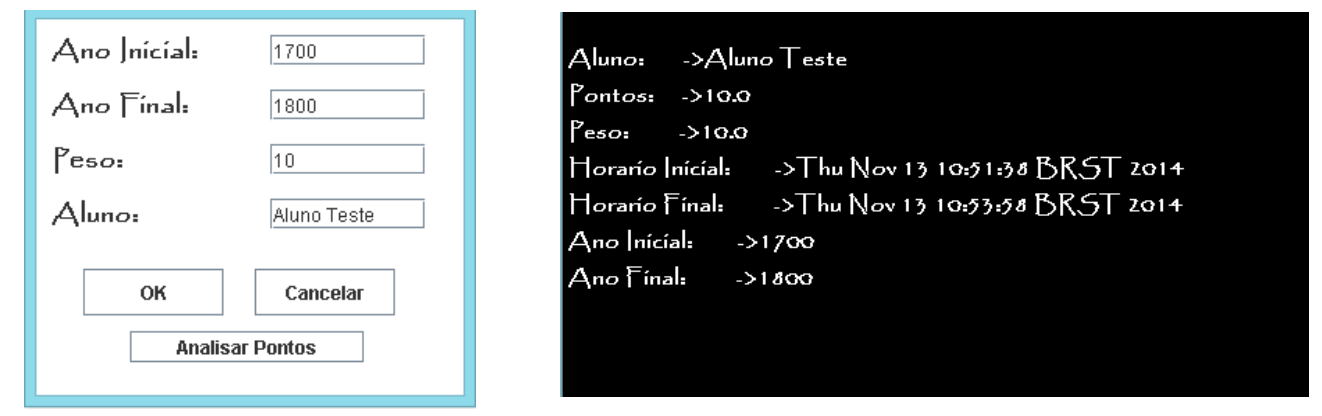

Figura 3 - (a) Tela inicial do OA na modalidade avaliação; (b) registro da pontuação obtida pelo usuário ao final da utilização do OA na modalidade avaliação.

Conforme pode ser visto na Figura 3, na tela de configuração da linha do tempo, há a opção "Analisar Pontos". Através desta opção o docente pode verificar qual foi a pontuação obtida pelo usuário na utilização do OA. Para isso, ele deve informar em uma 
nova tela as configurações utilizadas na avaliação e a hora de início e fim da atividade. Com isso, a avaliação pode ser aplicada para diferentes turmas em momentos diferentes e o resgate das pontuações se torna mais preciso (Figura 4).

\begin{tabular}{ll|} 
Ano Inicial: & 1700 \\
Ano Final: & 1800 \\
Peso: & 10 \\
Jnicio do Periodo (oo:Oo): & $10: 00$ \\
Fin do Periodo (oo:Oo): & $12: 00$ \\
Dia (o1/O1): & $13: 111$ \\
\hline OK & \\
\hline
\end{tabular}

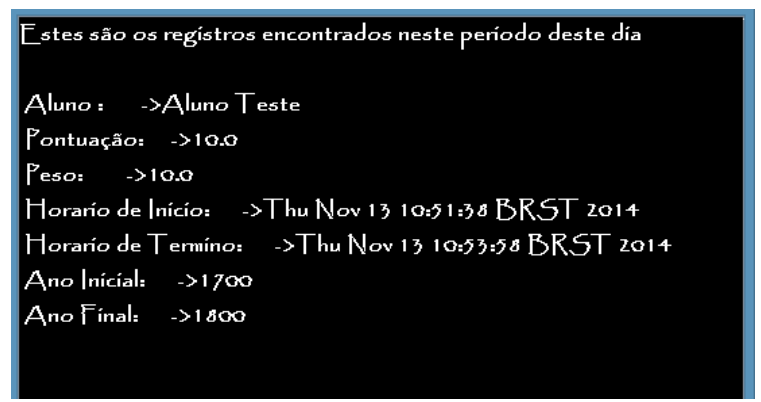

Figura 4 - (a) Tela que permite ao professor selecionar a atividade que deseja ver o resultado da avaliação; (b) registro da pontuação a partir da configuração selecionada pelo professor.

Com o objetivo de tornar o OA o mais reutilizável e dinâmico possível foi criado um módulo de administração. Como as informações sobre os pensadores e as contribuições são armazenadas em um arquivo XML, pensou-se em dar a opção para o docente alterar esse arquivo, inserindo, excluindo ou editando os pensadores disponíveis para utilização do OA através de uma interface gráfica. Assim, o OA pode ser utilizado para ilustrar diferentes conteúdos e em diferentes componentes curriculares.

\section{Análise e resultados}

A análise do OA foi realizada em duas turmas do $1^{\circ}$ ano do Ensino Médio. No total, 40 alunos utilizaram o recurso no modo atividade e avaliação e, em seguida, responderam a um questionário com o intuito de avaliar aspectos relacionados à usabilidade, ao layout e ao conteúdo do recurso. Estes estudantes já haviam visto em aula todos os conteúdos necessários para a realização dessa atividade, inclusive já havia sido realizado o exercício de elaboração de uma linha do tempo feita de forma manual, após a leitura de um livro sobre a história do surgimento da Sociologia. No entanto, alguns alunos apresentaram dificuldades para elaborar a linha do tempo, pois ela pressupunha ler o texto e identificar períodos históricos, acontecimentos e pensadores.

$\mathrm{Na}$ avaliação geral dos alunos foi mais fácil desenvolver esta atividade através do OA no que se refere à organização do pensamento em relação aos conteúdos. Também foi observado pelos estudantes que as duas atividades são de níveis diferentes e que a linha do tempo elaborada por eles exigiu mais estudo, mas foi mais confuso o processo de elaboração. No caso da linha do tempo digital, os estudantes julgaram ser esta mais eficaz para a organização do conhecimento. A Figura 5 indica que a maioria dos estudantes $(82,5 \%)$ consideraram que o OA facilitou a organização do conteúdo. 


\section{Quanto ao auxílio para organizar o pensamento sobre o conteúdo:}

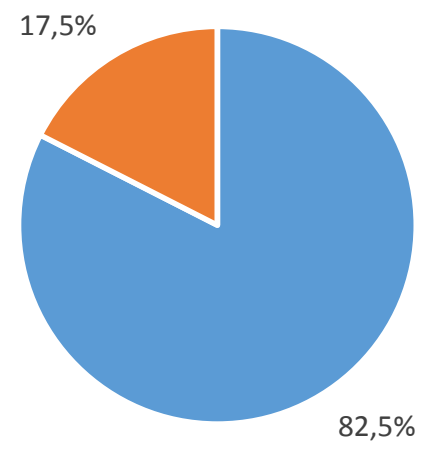

- Bom

- Regular

nuim

Figura 5 - Avaliação dos estudantes quanto ao auxílio dado pelo OA na elaboração de uma linha do tempo.

Em relação ao conteúdo disponibilizado na linha do tempo, 94,9\% dos estudantes julgaram estar adequado (Figura 6), o que significa que contemplou o que foi estudado em sala de aula. $\mathrm{O}$ fato do $\mathrm{OA}$ ser disponibilizado a outros professores com a possibilidade de alterar o conteúdo e incluir novas e/ou diferentes informações é um fator positivo, pois cada professor poderá ajustar o recurso de acordo com as necessidades da abordagem da sua disciplina, evitando que os estudantes tenham que responder sobre aspectos que ainda não foram estudados.

\section{Quanto à apresentação adequada do conteúdo:}

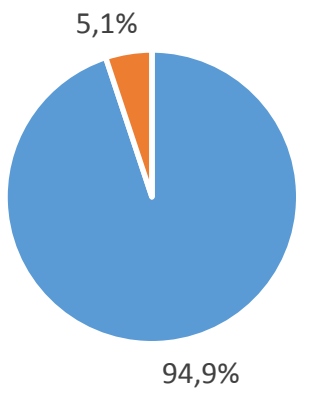

- Bom

- Regular

- Ruim

Figura 6 - Avaliação dos estudantes quanto ao conteúdo abordado pelo OA.

O layout do OA, no geral, foi bem avaliado. Algumas sugestões de melhoria foram feitas pelos estudantes no que se refere à necessidade de aumentar a fonte, pois eles indicaram a preferência por um layout mais colorido e não tão clássico quanto o proposto. Salienta-se que em relação ao aspecto específico da cor, trata-se de uma questão de preferências, não interferindo diretamente no uso do recurso. Também causou um desconforto em alguns estudantes o fato das imagens utilizadas não serem do mesmo tamanho. No entanto, essas imagens também se referem apenas à aparência do OA.

Obviamente, mesmo que se considere que esses aspectos não interferiram diretamente na usabilidade do OA eles podem ter algum impacto subjetivo durante o uso, 
por exemplo, um desconforto em relação à imagem geral que é apresentada e isso em última análise poderia não estimular o seu uso. Mas, por aquilo que a avaliação geral do OA permitiu identificar a rejeição ao layout não foi significativa a este ponto.

O uso da linha do tempo, segundo avaliação dos estudantes, ainda precisa ser aprimorado. A porcentagem de alunos que avaliou o quesito facilidade de uso da linha do tempo como regular e/ou ruim chegou a 47,5\% (Figura 7). As dificuldades de uso centraram-se especialmente no que se refere à movimentação das caixas, pois em algumas situações era difícil de mudá-las de lugar. Além disso, às vezes, uma caixa poderia ficar sobreposta à outra e, nesta situação, se o estudante não tivesse atento e não visse, teria dificuldades para identificar onde estava a caixa. Outro aspecto apontado pelos estudantes a ser repensado foi o tamanho da linha do tempo, que é grande tendo em vista que abrange pensadores de séculos diferentes. Uma alternativa a isso pode ser a redução da escala temporal ou ainda apresentar ao final do exercício uma versão compacta da linha do tempo, na qual seja possível visualizá-la de uma forma completa.

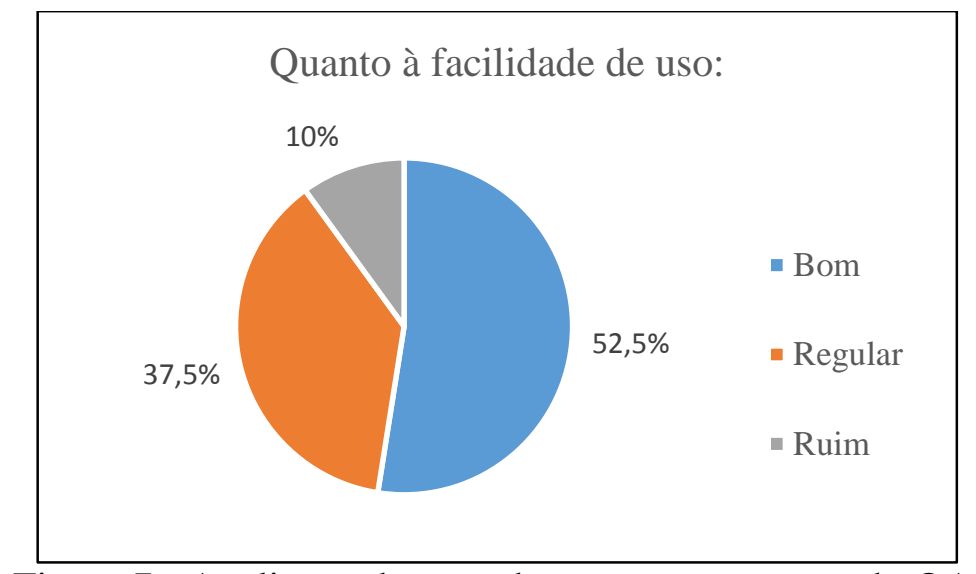

Figura 7 - Avaliação dos estudantes quanto ao uso do OA.

Em relação à utilização do OA no seu modo avaliação, a análise feita pela professora da disciplina foi de que ele não é prático, pois o resultado obtido pelos estudantes precisa ser coletado individualmente em cada um dos computadores utilizados pelos alunos e isso demanda um tempo considerável para turmas grandes. A respeito desta dificuldade existe a possibilidade de, futuramente, desenvolver o recurso na forma de uma aplicação para web, permitindo, com isso, facilitar a sua distribuição e coleta de informações.

A avaliação geral do OA mostrou que ele é um recurso interessante a ser utilizado pelos estudantes como uma maneira de exercitar um conjunto de informações estudadas, auxiliando na organização do conhecimento sobre a história do surgimento da Sociologia e os principais pensadores que contribuíram para isso. No que se refere à percepção do professor da disciplina, seu relato indicou que foi possível identificar o envolvimento dos estudantes para fazer a atividade prática. Isso se mostra importante, porque este é um conteúdo bastante teórico e alguns estudantes apresentam muitas dificuldades para relacionar pensadores com conceitos e com a sua contribuição para Sociologia em um determinado contexto histórico.

A professora da disciplina, através das observações feitas durante a realização da atividade e também nas suas aulas, identificou o envolvimento de alguns estudantes que em 
geral não se sentem muito atraídos pelas aulas ao desenvolver esta atividade da linha do tempo. Julga-se que isso se deve ao fato de a aula ter sido realizada em um laboratório de informática e porque os estudantes estavam em contato com um recurso digital, o que em geral é um fator de motivação para que os adolescentes desenvolvam alguma atividade. Obviamente, há aqui o entendimento de que este é apenas um recurso que pode facilitar o exercício de algum conteúdo, mas que não se coloca como uma possibilidade de substituir outras práticas importantes em sala de aula sem a utilização de tecnologias da informação.

\section{Considerações}

Este artigo apresentou um OA para a disciplina de Sociologia no Ensino Médio que consiste em uma linha do tempo interativa na qual devem ser relacionados pensadores clássicos da Sociologia às suas contribuições de forma cronológica. O OA foi desenvolvido na linguagem de programação Java e teve seus metadados descritos através do padrão OBAA - Lite.

Com o objetivo de verificar a recepção dos alunos ao OA, foi realizada uma etapa de validação com 40 alunos de uma turma de $1^{\circ}$ ano de Ensino Médio durante uma aula de Sociologia. Ao final da utilização os alunos responderam a um questionário sobre aspectos técnicos e sobre o conteúdo abordado pelo OA. Verificou-se que a maioria dos alunos aprovou o OA, tanto na parte técnica quanto na sua contribuição para compreensão do conteúdo. Em uma questão aberta do questionário, na qual os alunos poderiam fazer comentários dando sugestões, verificou-se que foram encontrados problemas relacionados a movimentação das caixas. Porém, foi possível perceber que o problema relatado não prejudicou a utilização do OA.

Além da percepção dos alunos, foi também analisada a percepção da docente que acompanhou os alunos durante a utilização do OA. Através de seus comentários foi possível identificar que o módulo de avaliação não é eficaz, tendo em vista que para recuperar a pontuação de cada usuário do OA é necessário verificar cada computador. Além disso, a docente também destacou pontos positivos, como o fato de que muitos alunos que normalmente não se interessam pela disciplina terem demonstrado bastante interesse em cumprir a tarefa.

Sendo assim, concluiu-se que o OA desenvolvido contribuiu para o ensino de Sociologia no nível médio, sendo um recurso interativo que auxilia na organização do pensamento abstrato abordado pela disciplina, sendo este aprovado pelos alunos e pela docente. Com o objetivo de compartilhar o recurso desenvolvido, ele está disponível no site http://www.vhflabs.com.br/projetos.html.

\section{Agradecimento}

Ao Instituto Federal de Educação, Ciência e Tecnologia do Rio Grande do Sul (IFRS) pelo fomento concedido ao projeto que originou este artigo.

\section{Referências bibliográficas}

BEZ, M.; VICARI, R. M.; SILVA, J. M. C.; RIBEIRO, A. M.; GLUZ, J. C.; PASSERINO, L. M; SANTOS, É. R.; PRIMO, T.; ROSSI, L ; BEHAR, P.; FILHO, R.; ROESLER, V. Proposta brasileira de metadados para objetos de aprendizagem baseados em agentes 
(OBAA). RENOTE - Revista Novas Tecnologias na Educação, Porto Alegre, v. 8, n. 2, p. 1-10, 2010.

BRASIL. Orientações Curriculares para o Ensino Médio. Ciências Humanas e suas Tecnologias. Secretaria de Educação Básica - Brasília: Ministério da Educação, Secretaria de Educação Básica, 2006.

LIEDKE FILHO, E. D. A sociologia no Brasil: história, teorias e desafios. Sociologias, Porto Alegre, ano 7, n. 14, p. 376-437, jul./dez. 2005.

MEDEIROS, L. F.; MUNHOZ, A. S. Materiais didáticos como objetos de aprendizagem: inovação pedagógica ou solução econômica? RENOTE - Revista Novas Tecnologias na Educação, Porto Alegre, v. 10, n. 3, p. 1-10, dez. 2012.

MILLS, W. C. A imaginação sociológica. Rio de Janeiro, Zahar, 1969. 246 p.

PEREIRA, L. H. Qualificando futuros professores de sociologia. Mediações, Londrina, v. 12, n. 1, p. 143-158, jan.-jun. 2007.

PEREIRA, L. H. Por uma sociologia da sociologia no Ensino Médio. In: CONGRESSO BRASILEIRO DE SOCIOLOGIA, 14, 2009, Rio de Janeiro. Anais. Rio de Janeiro: Sociedade Brasileira de Sociologia, 2009.

PEREIRA, T. I. Para além do senso comum: aportes para a construção do conhecimento sociológico na educação básica. In: CONGRESSO BRASILEIRO DE SOCIOLOGIA, 14, 2009, Rio de Janeiro. Anais. Rio de Janeiro: Sociedade Brasileira de Sociologia, 2009.

PIAGET, J. Desenvolvimento e aprendizagem. 1972. Disponível em: <https://ead.ufrgs.br/rooda/biblioteca/abrirArquivo.php/turmas/9276/materiais/10977.pdf > Acesso em: 12 nov. 2014.

SILVA, J. M. C. Análise técnica e pedagógica de metadados para objetos de aprendizagem. Porto Alegre: CINTED/UFRGS, 2011. 189p. Tese de Doutorado.

SILVEIRA, M. S.; CARNEIRO, M. L. F. Desconstruindo objetos de aprendizagem: reflexões sobre sua qualidade de uso. In: SIMPÓSIO BRASILEIRO DE INFORMÁTICA NA EDUCAÇÃO, 23, 2012, Rio de Janeiro. Anais. Rio de Janeiro: Sociedade Brasileira de Computação, 2012.

STEMPKOWSKI, I. F. A influência social na construção do conhecimento: a formação dos currículos de sociologia no ensino médio. Porto Alegre: PPGCS/PUCRS, 2010. 161 p. Dissertação de Mestrado.

WILEY, D. A. Learning object design and sequencing theory. Provo, Utah: Department Of Instructional Psychology And Technology/Brigham Young University, 2000. 131p. Tese de Doutorado. 